Proyecciones Journal of Mathematics

Vol. 34, No 3, pp. 205-218, September 2015.

Universidad Católica del Norte

Antofagasta - Chile

\title{
Lacunary I-convergent sequences of fuzzy real numbers
}

\author{
Binod Chandra Tripathy \\ Institute of Advanced Study in Science and Technology, India \\ and \\ Amar Jyoti Dutta \\ Pragjyotish College, India \\ Received : August 2013. Accepted : April 2015
}

\begin{abstract}
In this article we have studied on lacunary I-convergent sequences of fuzzy real numbers. We verify and establish some algebraic properties such as linearity, symmetric, convergence free etc. and also established some other results.
\end{abstract}

Key words and Phrases : Fuzzy real numbers; lacunary sequence; I-convergence; symmetric; convergence free; sequence algebra.

AMS Classification No : 40A05; 40A25; 40A30; 40C05. 


\section{Introduction}

The concept of fuzzy set was introduced by L.A. Zadeh in 1965. Later on different classes of sequences of fuzzy numbers have been introduced and studied by some researchers in the recent past. The notion of fuzzy numbers has been applied by the researchers for studying sequences spaces from different aspects. Recently works on sequences of fuzzy real numbers has been done by Altinok, Altin and Et [1], Altin, Et and Colak [2], Altin, Mursaleen and Altinok [3], Bilgin [4], Savas [11], Tripathy and Baruah ([14], [15], [16]), Tripathy and Borgohain [16], Tripathy and Dutta ([17], [18]), Tripathy and Sarma [24] and many others.

A fuzzy real number $X$ is a fuzzy set on $R$, more precisely a mapping $X: R \rightarrow I(=[0,1])$, associating each real number $t$ with its grade of membership $X(t)$, which satisfy the following conditions:

(i) $X$ is normal if there exists $t_{0} \in R$ such that $X\left(t_{0}\right)=1$.

(ii) $X$ upper-semi-continuous if for each $\varepsilon \geq 0, X^{-1}([0, a+\varepsilon))$, is open in the usual topology of $R$, for all $a \in I$.

(iii) $X$ is convex, if $X(t) \geq X(s) \cap X(r)=\min (X(s), X(r))$, where $s<t<r$.

The class of all upper-semi-continuous, normal, convex fuzzy real numbers is denoted by $R(I)$.

The set of real numbers $R$ can be embedded into $R(I)$, since each $r \in R$ can be regarded as a fuzzy number $\bar{r}$ given by

$$
\bar{r}(t)= \begin{cases}1 & \text { for } t=r \\ 0, & \text { for } t \neq r\end{cases}
$$

The additive identity and multiplicative identity of $R(I)$ are denoted by $\overline{0}$ and $\overline{1}$ respectively.

The $\alpha$-level set of a fuzzy real number $X$ is defined by

$$
[X]^{\alpha}= \begin{cases}\{t \in R: X(t) \geq \alpha\} & \text { for } 0<\alpha \leq 1, \\ c l\{t \in R: X(t)>\alpha\}, & \text { for } \alpha=0 .\end{cases}
$$

Let $D$ be the set of all closed bounded intervals $X=\left[X^{L}, X^{R}\right]$ then we write $X \leq Y$ if and only if $X^{L} \leq Y^{L}$ and $X^{R} \leq Y^{R}$. We can 
write $d(X, Y)=\max \left[X^{L}-Y^{L}, X^{R}-Y^{R}\right]$, where $X=\left[X^{L}, X^{R}\right]$ and $Y=\left[Y^{L}, Y^{R}\right]$. It is can be easily verify that $(D, d)$ is a complete metric space.

Consider the mapping $\bar{d}: R(I) \times R(I) \rightarrow R$ defined by

$$
\bar{d}(X, Y)=\sup _{0 \leq \alpha \leq 1} d\left(X^{\alpha}, Y^{\alpha}\right), \text { for } X, Y \in R(I) .
$$

Clearly $\bar{d}$ define a metric on $R(I)$ and $(R(I), \bar{d})$ is a complete metric space.

A fuzzy real-valued sequence $\left(X_{k}\right)$ is a function from the set of natural numbers into $R(I)$. It is denoted by $\left(X_{k}\right)$, where $X_{k} \in R(I)$, for all $k \in N$.

The set $E^{F}$ of sequences taken from $R(I)$ is closed under addition and scalar multiplication defined as follows:

Let $E^{F}$ be the class of sequence of fuzzy real numbers, the linearity of $E^{F}$ can be understand as follows:

For $\left(X_{k}\right),\left(Y_{k}\right) \in E^{F}$ and $r \in R$,

(i) $\left(X_{k}\right)+\left(Y_{k}\right)=\left(X_{k}+Y_{k}\right) \in E^{F}$

(ii) $r\left(X_{k}\right)=\left(r X_{k}\right) \in E^{F}$,

where

$$
r\left(X_{k}\right)(t)= \begin{cases}X_{k}\left(r^{-1} t\right), & \text { if } r \neq 0, \\ \overline{0}, & \text { if } r=0 .\end{cases}
$$

A sequence $\left(X_{k}\right)$ of fuzzy real numbers is said to be convergent to the fuzzy real number $X_{0}$, if for every $\varepsilon>0$, there exists $k_{0} \in N$ such that $\bar{d}\left(X_{k}, X_{0}\right)<\varepsilon$, for all $k \geq k_{0}$.

A sequence $\left(X_{k}\right)$ of fuzzy real numbers is said to be bounded if $\sup _{\mathrm{k}} \bar{d}\left(X_{k}, \overline{0}\right)<$ $\infty$, equivalently, if there exist $R^{*}(I)$ such that $\left|X_{k}\right| \leq \mu$ for all $n, k \in N$, where $R^{*}(I)$ denotes the set of all positive fuzzy real numbers.

\section{Definitions and Preliminaries}

A sequence $\left(X_{k}\right)$ of fuzzy real numbers is said to be convergent to the fuzzy real number $X_{0}$, if for every $\varepsilon>0$, there exists $k_{0} \in N$ such that 
$\bar{d}\left(X_{k}, X_{0}\right)<\varepsilon$ for all $k \geq k_{0}$. We denotes the set of sequences of fuzzy real numbers by $E^{F}$. We recall the following definitions:

Definition 2.1. A fuzzy real-valued sequence space $E^{F}$ is said to be normal (or solid) if $\left(X_{k}\right) \in E^{F}$, whenever $\left|X_{k}\right| \leq\left|Y_{k}\right|$ for all $k \in N$ and $\left(Y_{k}\right) \in E^{F}$.

Definition 2.2. A fuzzy real-valued sequence space $E^{F}$ is said to be symmetric if $\left(X_{\pi(k)}\right) \in E^{F}$, whenever $\left(X_{k}\right) \in E^{F}$, where $\pi$ is a permutation of $N$.

Definition 2.3. A fuzzy real-valued sequence space $E^{F}$ is said to be a sequence algebra if $\left(X_{k} \otimes Y_{k}\right) \in E^{F}$, whenever $\left(X_{k}\right),\left(Y_{k}\right) \in E^{F}$.

Definition 2.4. A fuzzy real-valued sequence space $E^{F}$ is said to be convergence free if $\left(X_{k}\right) \in E^{F}$ whenever $\left(Y_{k}\right) \in E^{F}$ and $Y_{k}=\overline{0}$ implies $X_{k}=\overline{0}$.

By a lacunary $\theta=\left(k_{r}\right)(r=0,1,2,3, \ldots \ldots)$ where $k_{0}=0$, we mean an increasing sequence of non-negative integers with $h_{r}=k_{r}-k_{r-1} \rightarrow \infty$ as $r \rightarrow \infty$. The interval determined by is given by $J_{r}=\left(k_{r-1}, k_{r}\right]$ and the ratio $\frac{k_{r}}{k_{r-1}}$ is denoted by $q_{r}$.

The space of lacunary strongly convergent sequence $N_{\theta}$ was defined by Freedman, Sember and Rapheal [6] as follows:

$$
N_{\theta}=\left\{x=\left(x_{k}\right): \lim _{r} \frac{1}{h_{r}} \sum_{k \in k_{r}}\left|x_{k}-L\right|, \text { for some } L\right\}
$$

The space of lacunary strongly convergent sequences, which is convergent to 0 is given by

$$
N_{\theta}^{0}=\left\{x=\left(x_{k}\right): \lim _{r} \frac{1}{h_{r}} \sum_{k \in J_{r}}\left|x_{k}\right|=0\right\}
$$

There is relation between the space $N_{\theta}$ and the space $\left|\sigma_{1}\right|$ of strongly Cesàro summable sequences. The space $\left|\sigma_{1}\right|$ is defined by

$$
\left|\sigma_{1}\right|=\left\{x=\left(x_{k}\right): \lim _{n \rightarrow \infty} \frac{1}{n} \sum_{k=1}^{n}\left|x_{k}-L\right|=0, \text { for some } L\right\}
$$


In special case when $\theta=\left(2^{r}\right), N_{\theta}=\left|\sigma_{1}\right|$. The concept of statistical convergence in terms of lacunary sequences was studied by Fridy and Orhan [5]. The classes of lacunary fuzzy sequences was studied by Altinok, Altin and Et.[1], Altin, Et. and Colak [2], Bligin [4], Nuray [8], Tripathy and Dutta [26] and others.

The notion of $I$-convergence of real valued sequence was studied by Kostyrko, Salát and Wilczyński [7], Salat, Tripathy and Ziman ([9], [10]), Tripathy and Hazarika ([19], [20], [21]), Tripathy and Mahanta [22] and many others.

Definition 2.5. Let $X$ be a non-empty set, then a non-void class $I \subset P(X)$ (power set of $X$ ) is called an ideal if (i) $\Phi \in I$, (ii) $I$ is additive i.e. $A, B \in$ $I \subset A \cup B \in I$ and (iii) $I$ is hereditary i.e. $A \in I$ and $B \subset A \Rightarrow B \in I$.

An ideal $I$ is said to be non-trivial if $I \neq \Phi$ and $X \notin I$.

Definition 2.6. A non-trivial ideal $I$ is said to be admissible if $I$ contains all singleton subsets of $X$ i.e. $\{x\} \in I$ for every $x \in X$.

Definition 2.7. A non-trivial ideal $I$ is said to be maximal if there does not exist any non-trivial ideal $J \neq I$ containing $I$ as a subset.

Definition 2.8. Let $X$ be a non-empty set then a non-void class $F \subset$ $P(X)$ is said to be a filter on $X$ if and only if $(i) \emptyset \notin F$; (ii) For each $A, B \in F \Rightarrow A \cap B \in F$ and $(i i i) A \in F, A \subset B \Rightarrow B \subset F$. For any ideal $I$ there is a filter $F(I)$ corresponding to $I$ given by

$$
F(I)=\{K \subset N: N \backslash K \in I\} .
$$

Some particular cases of $I$ - convergence of sequences are as follows:

(a) If we take $I=I_{f}=\{A \subset N: A$ is a finite subset $\}$. Then $I_{f}$ is a non-trivial admissible ideal and the corresponding convergence is the usual convergence of sequences.

(b) If we take $I=I_{\delta}=\{A \subset N: \delta(A)=0\}$, where $\delta(A)$ denote the asymptotic (natural) density of the set, defined by $\delta(A)=\lim _{n} \frac{1}{n} \mid\{k \leq n$ : $k \in A\} \mid$, where the vertical bars indicate the cardinality of the set. Then 
$I_{\delta}$ is a non-trivial admissible ideal and the corresponding convergence is known as statistical convergence.

The notion of statistical convergence has been investigated from different aspects and matrix classes have been characterized by Fridy and Orhan [5], Altin, Mursaleen and Altinok [3], Tripathy ([12], [13]), Tripathy and Baruah [15], Tripathy and Sarma [23], Tripathy and Sen [25] and others.

(c) If we take $I=I_{d}=\{A \subset N: d(A)=0\}$, where $d(A)$ denote the logarithmic density of the set $A$, defined by $d(A)=\lim _{n} \frac{1}{s_{n}} \sum_{k=1}^{n} \frac{\chi_{A}(k)}{k}$, where $s_{k}=\sum_{k=1}^{n} \frac{1}{k}$. Then is a non-trivial admissible ideal and the corresponding convergence as the logarithmic convergence of sequences.

Let $\theta=\left(k_{r}\right)$ be a lacunary sequence. Then a sequence $\left(x_{k}\right)$ is said to be lacunary $I$-convergent to $L$ if for every $\varepsilon>0,\left\{r \in N: h_{r}^{-1} \sum_{k \in J_{r}}\left|x_{k}-L\right| \geq \varepsilon\right\} \in$ $I$. We write it as $I_{\theta}-\lim x_{k}=L$.

Let $\theta=\left(k_{r}\right)$ be a lacunary sequence. Then a sequence $\left(x_{k}\right)$ is said to be lacunary $I$-null if $L=0$. We write it as $I_{\theta}-\lim x_{k}=0$.

Let $\theta=\left(k_{r}\right)$ be a lacunary sequence. Then a sequence $\left(x_{k}\right)$ is said to be lacunary $I$-Cauchy if there is a subsequence $\left(x_{k_{r}}\right)$ of $\left(x_{k}\right)$ such that $k_{r} \in I_{r}$, for each $r$ and for every $\varepsilon>0$ such that $\left\{r \in N: h_{r}^{-1} \sum_{k \in J_{r}}\left|x_{k}-x_{k_{r}}\right| \geq \varepsilon\right\} \in$ $I$.

A lacunary sequence $\dot{\theta}=\left(\bar{k}_{r}^{\prime}\right)$ is said to be a lacunary refinement of the lacunary sequence $\theta=\left(k_{r}\right)$ if $\left(k_{r}\right) \subset\left(k_{r}^{\prime}\right)$.

We defined the above definitions for fuzzy real-valued sequences.

Definition 2.9. Let $\theta=\left(k_{r}\right)$ be a lacunary sequence. Then a sequence $\left(X_{r}\right)$ of fuzzy real numbers is said to be lacunary $I$-convergent to a fuzzy real number $L$ if for every $\varepsilon>0$, such that 


$$
\left\{r \in N: h_{r}^{-1} \sum_{k \in k_{r}} \bar{d}\left(X_{k}, L\right) \geq \varepsilon\right\} \in I .
$$

Definition 2.10. Let $\theta=\left(k_{r}\right)$ be a lacunary sequence. Then a sequence $\left(X_{k}\right)$ of fuzzy real numbers is said to be lacunary $I$-Cauchy if there is a subsequence $\left(X_{k_{r}}\right)$ of $\left(X_{k}\right)$ such that $k_{r} \in I_{r}$, for each $r$ and for every $\varepsilon>0$ such that

$$
\left\{r \in N: h_{r}^{-1} \sum_{k \in k_{r}} \bar{d}\left(X_{k}, X_{k_{r}}\right) \geq \varepsilon\right\} \in I .
$$

Throughout $\left(c_{0}^{I}\right)_{\theta}^{F}$ and $\left(c^{I}\right)_{\theta}^{F}$ will represents respectively the sets of lacunary $I$-null and lacunary $I$ - convergent sequences of fuzzy real numbers and $I$ is neither maximal nor finite.

\section{Main results}

Theorem 3.1. Let $\left(X_{k}\right)$ and $\left(Y_{k}\right)$ be sequences of fuzzy real numbers. Then

(a) If $I_{\theta}-\lim X_{k}=X_{0}$ then $I_{\theta}-\lim c X_{k}=c X_{0}$, for $c \in R$.

(b) If $I_{\theta}-\lim X_{k}=X_{0}$ and If $I_{\theta}-\lim Y_{k}=Y_{0}$, then $I_{\theta}-\lim \left(X_{k}+Y_{k}\right)=$ $X_{0}+Y_{0}$.

Proof. (a) Let $I_{\theta}-\lim X_{k}=X_{0}$ and $X_{k}^{\alpha}$ denote the $\alpha$-level set of $X_{k}$, where $\alpha \in[0,1]$.

Since $d\left(c X_{k}^{\alpha}, c X_{0}^{\alpha}\right)=|c| d\left(X_{k}^{\alpha}, X_{0}^{\alpha}\right)$, for $c \in R$.

$\Rightarrow \sup _{\alpha} d\left(c X_{k}^{\alpha}, c X_{0}^{\alpha}\right)=|c|{ }_{\alpha}^{\sup } d\left(X_{k}^{\alpha}, X_{0}^{\alpha}\right)$.

$\Rightarrow \bar{d}\left(c X_{k}, c X_{0}\right)=|c| \bar{d}\left(X_{k}, X_{0}\right)$.

Now for a given $\varepsilon>0$.

$$
\left|\left\{r \in N: \frac{1}{h_{r}} \sum_{k \in J_{r}} \bar{d}\left(c X_{k}, c X_{0}\right) \geq \varepsilon\right\}\right| \leq\left|\left\{r \in N: \frac{1}{h_{r}} \sum_{k \in J_{r}} \bar{d}\left(X_{k}, X_{0}\right) \geq \frac{\varepsilon}{|c|}\right\}\right| .
$$

Hence it follows that $I_{\theta}-\lim c X_{k}=c X_{0}$. 
(b)Let $I_{\theta}-\lim X_{k}=X_{0}, I_{\theta}-\lim Y_{k}=Y_{0}$ and $X_{k}^{\alpha}$ denote the $\alpha$-level set of $X_{k}$, where $\alpha \in[0,1]$.

Then we have

$$
\begin{aligned}
& d\left(X_{k}^{\alpha}+Y_{k}^{\alpha}, X_{0}^{\alpha}+Y_{0}^{\alpha}\right) \leq d\left(X_{k}^{\alpha}, X_{0}^{\alpha}\right)+d\left(Y_{k}^{\alpha}, Y_{0}^{\alpha}\right) \\
& \Rightarrow \sup _{\alpha} d\left(\begin{array}{l}
\alpha \\
k
\end{array} Y_{k}^{\alpha}, X_{0}^{\alpha}+Y_{0}^{\alpha}\right) \leq \sup _{\alpha} d\left(X_{k}^{\alpha}, X_{0}^{\alpha}\right)+d\left(Y_{k}^{\alpha}, Y_{0}^{\alpha}\right) \\
& \Rightarrow \bar{d}\left(X_{k}^{\alpha}+Y_{k}^{\alpha}, X_{0}^{\alpha}+Y_{0}^{\alpha}\right) \leq \bar{d}\left(X_{k}^{\alpha}, X_{0}^{\alpha}\right)+\bar{d}\left(Y_{k}^{\alpha}, Y_{0}^{\alpha}\right) .
\end{aligned}
$$

For a given $\varepsilon>0$, we have,

$$
\begin{gathered}
\left|\left\{r \in N: \frac{1}{h_{r}} \sum_{k \in J_{r}} \bar{d}\left(X_{k}+Y_{k}, X_{0}+Y_{0}\right) \geq \varepsilon\right\}\right| . \\
\leq\left|\left\{r \in N: \frac{1}{h_{r}}\left(\sum_{k \in J_{r}} \bar{d}\left(X_{k}, X_{0}\right)+\sum_{k \in J_{r}} \bar{d}\left(Y_{k}, Y_{0}\right) \geq \varepsilon\right)\right\}\right| . \\
\leq\left|\left\{r \in N: \frac{1}{h_{r}} \sum_{k \in J_{r}} \bar{d}\left(X_{k}, X_{0}\right) \geq \frac{\varepsilon}{2}\right\}\right|+\left|\left\{r \in N: \frac{1}{h_{r}} \sum_{k \in J_{r}} \bar{d}\left(Y_{k}, Y_{0}\right) \geq \frac{\varepsilon}{2}\right\}\right| .
\end{gathered}
$$

Hence it follows that $I_{\theta}-\lim \left(X_{k}+Y_{k}\right)=X_{0}+Y_{0}$.

Theorem 3.2. Let $\left(X_{k}\right),\left(Y_{k}\right)$ and $\left(Z_{k}\right)$ be fuzzy real valued sequences such that

$(i)\left(X_{k}\right) \leq\left(Y_{k}\right) \leq\left(Z_{k}\right)$,

(ii) $I_{\theta}-\lim X_{k}=I_{\theta}-\lim Z_{k}=L$.

Then $I_{\theta}-\lim Y_{k}=L$.

Proof. Since $I_{\theta}-\lim X_{k}=L$ and $I_{\theta}-\lim Z_{k}=L$, for a chosen $\varepsilon>0$,

we have

$$
\left\{r \in N: \frac{1}{h_{r}} \sum \bar{d}\left(X_{k}, L\right) \geq \varepsilon\right\} \in I
$$


and

$$
\left\{r \in N: \frac{1}{h_{r}} \sum \bar{d}\left(Z_{k}, L\right) \geq \varepsilon\right\} \in I .
$$

Then the sets $A=\left\{r \in N: \frac{1}{h_{r}} \sum \bar{d}\left(X_{k}, L\right)<\varepsilon\right\}$ and $B=\left\{r \in N: \frac{1}{h_{r}} \sum \bar{d}\left(Z_{k}, L\right)<\varepsilon\right\}$ contained in the filter $F(I)$. Now consider the set $C=\left\{r \in N: \frac{1}{h_{r}} \sum \bar{d}\left(Y_{k}, L\right)<\varepsilon\right\}$. It is clear that $C \supseteq A \cap B$ and since $A \cap B \in F(I) \Rightarrow C \in F(I)$. Thus $C=\left\{r \in N: \frac{1}{h_{r}} \sum \bar{d}\left(Y_{k}, L\right) \geq \varepsilon\right\} \in$ $I$. This completes the proof.

Theorem 3.3. If $\dot{\theta}$ is a lacunary refinement of $\theta$ and $I_{\theta^{\prime}}-\lim X_{k}=X_{0}$ then $I_{\theta}-\lim X_{k}=X_{0}$.

Proof. Suppose each $J_{r}\left(=\left(k_{r-1}, k_{r}\right]\right)$ of $\theta$ contains a point $\left\{k_{r, i}^{\prime}\right\}_{r=1}^{v(r)}$ of $\dot{\theta}$, where $v(r) \geq 1$ for all $r$ such that

$$
k_{r-1}<k_{r, 1}^{\prime}<k_{r, 2}^{\prime}<----<k_{r, v(r)}^{\prime}=k_{r}
$$

where $J_{r, i}^{\prime}=\left(k_{r, r-1}^{\prime}, k_{r, i}^{\prime}\right]$. Let $\left\{J_{i}^{*}\right\}_{i=1}^{\infty}$ be the sequence of intervals $\left\{J_{r, i}^{\prime}\right\}$ ordered by increasing right ends points. Since $I_{\dot{\theta}}-\lim X_{k}=X_{0}$, for a given $\varepsilon>0$, we have, $\left\{i \in N:{\frac{1}{h_{i}}}^{*} \sum_{J_{i}^{*} \subset J_{r}} \bar{d}\left(X_{k}, X_{0}\right) \geq \varepsilon\right\} \in I, \ldots-$ - (1) where $h_{r}=k_{r}-k_{r-1}, h_{r, i}^{\prime}=k_{r, i}^{\prime}-k_{r, i-1}^{\prime}$ and $h_{r, 1}^{\prime}=k_{r, 1}^{\prime}-k_{r-1,1}^{\prime}$. Now for each $\varepsilon>0$, we have $\left\{r \in N: \frac{1}{h_{r}} \sum \bar{d}\left(X_{k}, X_{0}\right)<\varepsilon\right\} \subset\left\{i \in N: \frac{1}{h_{i}^{*}} \sum \bar{d}\left(X_{k}, X_{0}\right)<\varepsilon\right\} \in I$, [using (1)].

Hence $I_{\theta}-\lim X_{k}=X_{0}$.

This completes the proof.

Theorem 3.4. The classes of sequence $\left(c_{0}^{I}\right)_{\theta}^{F},\left(c^{I}\right)_{\theta}^{F}$ are not symmetric in general.

Proof. The proof follows from the following example: 
Example 3.1. Let $I \neq I_{f}$ and $\theta=\left(3^{r}\right)$ be a lacunary sequence. Consider $\left(X_{k}\right) \in Z$, where $Z=\left(c_{0}^{I}\right)_{\theta}^{F},\left(c^{I}\right)_{\theta}^{F}$ defined as follows:

For $k=i^{2}, i \in N$,

$$
\begin{aligned}
& r\left(X_{k}\right)(t)= \begin{cases}0, & \text { for } t \leq-\sqrt{k}, \\
-k^{-1} t^{2}+1, & \text { for }-\sqrt{k} \leq t \leq \sqrt{k}, \\
0, & \text { for } t \geq \sqrt{k} .\end{cases} \\
& X_{k}=\overline{0}, \text { for } k \neq i^{2}, i \in N .
\end{aligned}
$$

Then $\left(X_{k}\right) \in Z$, for $Z=\left(c_{0}^{I}\right)_{\theta}^{F},\left(c^{I}\right)_{\theta}^{F}$.

Now consider the rearrangement $\left(Y_{k}\right)$ of $\left(X_{k}\right)$ defined as follows

$$
\begin{gathered}
Y_{k}(t)=X_{k}(t), \text { for } k \text { even, } \\
Y_{k}=\overline{0}, \text { otherwise. }
\end{gathered}
$$

We observe that $\left(Y_{k}\right) \notin Z$, for $Z=\left(c_{0}^{I}\right)_{\theta}^{F},\left(c^{I}\right)_{\theta}^{F}$. This completes the proof.

Theorem 3.5. The classes of sequence $\left(c_{0}^{I}\right)_{\theta}^{F},\left(c^{I}\right)_{\theta}^{F}$ are not convergence free in general.

Proof. The proof follows from the following example.

Example 3.2. Let $I \neq I_{f}$ and $\theta=\left(3^{r}\right)$ be a lacunary sequence. Consider the fuzzy real-valued sequence $\left(X_{k}\right)$ defined as follows:

For $k \notin i^{2}, i \in N$,

$$
\left(X_{k}\right)(t)= \begin{cases}0, & \text { for } t \leq-k^{-1} \\ \left\{-(k t)^{2}+1\right\}, & \text { for }-k^{-1} \leq t \leq k^{-1} \\ 0, & \text { for } t \geq k^{-1}\end{cases}
$$

and

$$
X_{k}=\overline{0}, \text { for } k=i^{2}, i \in N .
$$

It is clear that $\left(X_{k}\right) \in Z$, for $Z=\left(c_{0}^{I}\right)_{\theta}^{F},\left(c^{I}\right)_{\theta}^{F}$. 
Now consider the sequence $\left(Y_{k}\right)$ defined as follows:

For $k \neq i^{2}, i \in N$,

$$
\begin{aligned}
\left(Y_{k}\right)(t) & = \begin{cases}0, & \text { for } t \leq-k^{2}, \\
\left\{-k^{-4} t^{2}+1\right\}, & \text { for }-k^{2} \leq t \leq k^{2}, \\
0, & \text { for } t \geq k^{2}\end{cases} \\
Y_{k} & =\overline{0}, \text { for } k=i^{2}, i \in N
\end{aligned}
$$

It is clear that $\left(Y_{k}\right) \notin Z$, for $Z=\left(c_{0}^{I}\right)_{\theta}^{F},\left(c^{I}\right)_{\theta}^{F}$. Hence $\left(c_{0}^{I}\right)_{\theta}^{F},\left(c^{I}\right)_{\theta}^{F}$ are not convergence free in general. This completes the proof.

Theorem 3.6. The classes of sequence $\left(c_{0}^{I}\right)_{\theta}^{F},\left(c^{I}\right)_{\theta}^{F}$ are not sequence algebra in general.

Proof. We shall prove for the space $\left(c^{I}\right)_{\theta}^{F}$ and for the other space it can be prove similarly. The proof follows from the following example.

Example 3.3. Let $I \neq I_{f}$ and $\theta=\left(3^{r}\right)$ be a lacunary sequence.

Consider the sequences $\left(X_{k}\right),\left(Y_{k}\right)$ defined as follows

For $k=i^{2}, i \in N$,

$$
\begin{aligned}
& \left(X_{k}\right)(t)= \begin{cases}1+t k^{-\frac{1}{2}}, & \text { for }-\sqrt{k} \leq t \leq 0, \\
1-t k^{-\frac{1}{2}}, & \text { for } 0 \leq t \leq \sqrt{k}, \\
0, & \text { otherwise }\end{cases} \\
& X_{k}=\overline{0} \text { for } k \neq i^{2}, i \in N \\
& \left(Y_{k}\right)(t)= \begin{cases}1+t k^{-\frac{1}{2}}, & \text { for }-\sqrt{k} \leq t \leq 0, \\
1-t k^{-\frac{1}{2}}, & \text { for } 0 \leq t \leq \sqrt{k}, \\
0, & \text { otherwise. }\end{cases} \\
& Y_{k}=\overline{1} \text { for } k \neq i^{2}, i \in N .
\end{aligned}
$$

We observe that $\left(X_{k}\right),\left(Y_{k}\right) \in\left(c^{I}\right)_{\theta}^{F}$ and $\left(X_{k} \otimes Y_{k}\right) \notin\left(c^{I}\right)_{\theta}^{F}$. This completes the proof. 


\section{References}

[1] H. Altmok, Y. Altin, M. Et, Lacunary almost statistical convergence of fuzzy numbers, Thai J. Math., 2 (2), pp. 265-274, (2004).

[2] Y. Altin, M. Et, R. Colak, Lacunary statistical and lacunary strongly convergence of generalized difference sequences of fuzzy numbers, Comput. Math. Appl., 52, pp. 1011-1020, (2006).

[3] Y. Altin, M. Mursaleen, H. Altinok, Statistical summability $(C, 1)$ for sequences of fuzzy real numbers and a Tauberian theorem, J. Intelligent Fuzzy Systems, 21 (6), pp. 379-384, (2010).

[4] T. Bligin, Lacunary strongly $\Delta$-convergent sequences of fuzzy numbers, Inf. Sci., 160 (1-4), pp. 201-206, (2004).

[5] J. A. Friday, C. Orhan, Lacunary statistical convergence; Pacific J. Math., 160 (1), pp. 43-51, (1993).

[6] A. R. Freedman, J. J. Sember, M. Raphael, Some Cesàro type summability spaces, Proc. London. Math. Soc., 37, pp. 508-520, (1978).

[7] P. Kostyrko, T. Šalăt, W. Wilczynski, I-convergence, Real Anal. Exchange, 26 (2), pp. 669-686, (2000-2001).

[8] F. Nuray, Lacunary statistical convergence of sequences of fuzzy numbers, Fuzzy Sets Systems, 99, pp. 353-356, (1998).

[9] T. Šalăt, B. C. Tripathy, M. Ziman, On some properties of $I$ convergence, Tatra Mt. Math. Publ., 28 , pp. 279-286, (2004).

[10] T. Šalăt, B. C. Tripathy, M. Ziman, On I-convergence field, Italian J. Pure Appl., Math. 17, pp. 45-54, (2005).

[11] E. Savas, New double sequence spaces of fuzzy numbers, Quaestiones Mathematicae, 33, pp. 449-456, (2010).

[12] B. C. Tripathy, Matrix transformation between some classes of sequences, J. Math. Anal. Appl., 206, pp. 448-450, (1997).

[13] B. C. Tripathy, On generalized difference paranormed statistically convergent sequences, Indian J. Pure Appl. Math. 35(5), pp. 655-663, (2004). 
[14] B. C. Tripathy, A. Baruah, New type of difference sequence spaces of fuzzy real numbers, Math. Modelling. Anal., 14 (3), pp. 391-397, (2009).

[15] B. C. Tripathy, A. Baruah, Lacunary statistically convergent and lacunary strongly convergent generalized difference sequences of fuzzy real numbers, Kyungpook Math. J., 50, pp. 565-574, (2010).

[16] B. C. Tripathy, S. Borgogain, The sequence space $m\left(M, \phi, \Delta_{m}^{n}, p\right)^{F}$, Math. Modelling Anal., 13 (4), pp. 577-586, (2008).

[17] B. C. Tripathy, A. J. Dutta, On fuzzy real-valued double sequence spaces ${ }_{2} \ell_{F}^{p}$, Math. Comput. Modelling, 46 (9-10), pp. 1294-1299, (2007).

[18] B. C. Tripathy, A. J. Dutta, Bounded variation double sequence space of fuzzy real numbers, Comput. Math. Appl., 59 (2), pp. 1031-1037, (2010).

[19] B. C. Tripathy, B. Hazarika, I-convergent sequence spaces associated with multiplier sequence spaces, Math. Ineq. Appl., 11 (3), pp. 543$548,(2008)$.

[20] B. C. Tripathy, B. Hazarika, Paranormed I-convergent sequences spaces, Math. Slovaca, 59 (4), pp. 485-494, (2009).

[21] B. C. Tripathy, B. Hazarika, I-convergent sequences spaces defined by Orlicz function, Acta Math. Appl. Sin. (Engl. Ser.), 27 (1), pp. 149-154, (2011).

[22] B. C. Tripathy, S. Mahanta, On $I$-acceleration convergence of sequences, J. Franklin Inst., 347, pp. 591-598, (2010).

[23] B. C. Tripathy, B. Sarma, Statistically convergent difference double sequence spaces, Acta Math. Sin., 24 (5), pp. 737-742, (2008).

[24] B. C. Tripathy, B. Sarma, Sequence spaces of fuzzy real numbers defined by Orlicz functions, Math. Slovaca, 58 (5), pp. 621-628, (2008).

[25] B. C. Tripathy, M. Sen, On generalized statistically convergent sequences, Indian J. Pure Appl. Math., 32 (11), pp. 1689-1694, (2001).

[26] B. C. Tripathy, A. J. Dutta, Lacunary bounded variation sequence of fuzzy real numbers, J. Intelligent Fuzzy Systems, 24, pp. 185-189, (2013). 


\section{Binod Chandra Tripathy}

Mathematical Sciences Division

Institute of Advanced Study in Science and Technology

Paschim Baragaon, Garchuk, Guwahati-781035

India

e-mail : tripathybc@yahoo.com

and

\section{Amar Jyoti Dutta}

Department of Mathematics

Pragjyotish College

Guwahati-781009, Assam

India

e-mail : amar_iasst@yahoo.co.in 